\title{
Incorporation of Nutritional Factors and Insulin Resistance into Insulin Sliding Scales in the Non-Critical Care Inpatient Setting: A "Before/After" Study
}

\section{Philipp Schuetz ${ }^{* \#, ~ N i n a ~ B r a u n ", ~ S a r a ~ G e r m a n n \#, ~ A n n i c ~ B a u m g a r t n e r ~ a n d ~ B e a t ~ M u e l l e r ~}$}

Medical University Department, Division of Endocrinology, Kantonsspital Aarau, Switzerland

"Authors equally contributed

\begin{abstract}
Objective: Current guidelines emphasise the importance of effective glucose control in medical inpatients outside critical care. Traditional glucose-adapted insulin sliding scales (SSCs) may inaccurately estimate insulin requirements, resulting in hypoglycaemia, hyperglycaemia, or both. We retrospectively performed a "before/after" analysis investigating whether an SSC also incorporating carbohydrate intake and estimated insulin resistance (iSSC) improves glucose control relative to that with conventional SSCs.

Methods: We compared glucose control during the initial 120 inpatient hours in patient groups with hospital diagnoses of lower respiratory tract infection or an acute cardiac condition and diabetes mellitus as comorbidity, one group treated during 2010, after introduction of an iSSC, and an historical control group treated during 2008 , with an SSC as the standard of care. Mean glucose levels, hypoglycaemic and hyperglycaemic episodes, glucose variability, and hospital outcomes (length-of-stay, all-cause mortality, intensive care unit admission) were compared using multivariate linear regression analysis adjusted for potentially important confounders.

Results: Of 215 included patients, 59.5\% ( $n=128)$ were in the iSSC group, 40.5\% $(n=87)$ in the historical control group. Relative to controls, iSSC patients had consistently greater probability of effective glucose control and better hospital outcomes; however, these differences were insufficiently powered to attain statistical significance $(p \geq 0.114)$.

Conclusions: Our results suggest that incorporating nutritional factors and insulin resistance into an SSC may improve glucose control and clinical outcomes in the everyday non-critical care inpatient setting. Due to the small patient sample and borderline significant results, we endorse further large, prospective, randomised controlled studies conclusively answering this question.
\end{abstract}

Keywords: Diabetes mellitus; Glucose control; Insulin; Sliding scale; Hospital; Inpatient; Outcomes

\section{Introduction}

Recent studies support the association of hyperglycaemia and adverse clinical outcomes in hospitalised patients across different medical diagnoses and settings [1-10]. Although most of the literature originates from critical care units, the association of increased glucose levels and adverse hospital outcomes also may apply to non-critical care medical wards. Therefore recently updated Endocrine Society guidelines state that hypergylcaemia is a common, serious and costly health care problem in hospitalised patients and should be prevented or corrected [11]. Implementing a standardised subcutaneous (s.c.) insulin order set promoting and guiding the use of scheduled basal and nutritional insulin therapy is a key intervention in inpatient diabetes management.

Unfortunately, inpatient s.c. insulin protocols are not well defined outside critical care. Few studies on insulin regimens to improve glycaemic control in medical ward patients have been published [1214]. Moreover, the reported studies were limited, either by focusing only on body mass index (BMI) to estimate required insulin dosing, by using since-superseded, relatively high target blood glucose levels of 5.6-10.0 $\mathrm{mmol} / \mathrm{L}$, or by having retrospective designs [15-19]. Importantly, not only BMI and the degree of visceral obesity, but also other factors such as acute illness-associated inflammation and stress hormone release may enhance insulin resistance [20-26]. Therefore, insulin requirements in hospitalised patients will likely vary, with important differences within and between individuals, depending on underlying medical conditions. The extent of insulin resistance is usually unknown upon admission, and insulin requirements may change rapidly during hospitalisation.
Based on these considerations, our research group recently developed an "improved" sliding scale insulin algorithm (iSSC) incorporating key individual characteristics for each patient, including his or her acute medical condition [27]. The protocol's goal was to define s.c. insulin requirements in order to rapidly achieve and consistently maintain target blood glucose levels [27]. In a prospective randomised controlled trial at two Swiss hospitals [27], patients cared for using the algorithm showed faster, longer-lasting hyperglycaemia correction than did controls managed with a sliding scale insulin protocol adapted only to glucose concentration (SSC). In 2010, a third Swiss institution, Kantonsspital Aarau (Cantonal Hospital of Aarau), which had not been a site of the randomised controlled study, introduced a slightly modified version of the iSSC for all inpatients with diabetes mellitus and or hyperglycemia treated in non-critical care medical wards. The aim of the current "before/after" study was to assess safety and efficacy of the

*Corresponding author: Philipp Schuetz, Medical University Department, Kantonsspital Aarau, Tellstrasse, CH-5001 Aarau, Switzerland, Tel: +41 (0)62 838 9524; Fax: +41 (0)62 838 9525; E-mail: Schuetzph@gmail.com

Received December 18, 2013; Accepted January 14, 2014; Published January 19, 2014

Citation: Schuetz P, Braun N, Germann S, Baumgartner A, Mueller B (2014) Incorporation of Nutritional Factors and Insulin Resistance into Insulin Sliding Scales in the Non-Critical Care Inpatient Setting: A "Before/After" Study. J Diabetes Metab 2: 325. doi:10.4172/2155-6156.1000325

Copyright: (c) 2014 Schuetz P, et al. This is an open-access article distributed under the terms of the Creative Commons Attribution License, which permits unrestricted use, distribution, and reproduction in any medium, provided the original author and source are credited. 
iSSC in everyday clinical practice at Kantonsspital Aarau, by comparing outcomes in patients treated post-algorithm adoption versus those in patients treated when an SSC was the routine insulin protocol.

\section{Methods}

\section{Patients and ethics}

This retrospective study compared consecutive eligible medical ward inpatients with type 1 or type 2 diabetes mellitus as a comorbidity who were treated with the iSSC from 1 January-31 December 2010 against those managed with an SSC, the then-current standard of care in the hospital, from 1 January-31 December 2008 (historical controls). Internal medicine physicians and nurses had been trained regarding the iSSC, and the protocol had been introduced, over the course of 2009.

Patients were selected via the hospital's electronic medical record system, and data for this analysis were collected by hand searching medical histories of all eligible individuals. To reduce patient heterogeneity, the cause of hospitalisation had to be either 1) a lower respiratory tract infection (LRTI) including pneumonia or chronic obstructive pulmonary disease exacerbation, or 2) an acute cardiac condition including acute coronary heart disease (ST-elevation or non-ST-elevation myocardial infarction, unstable angina pectoris) or acute heart failure. Diagnosis of LRTI or acute cardiac disease as the cause of admission and of diabetes as a comorbidity was identified based on medical records.

Diabetes could not have been first diagnosed at or after the index admission, or have been the primary cause of hospitalisation. Additionally, we excluded patients if (a) the iSSC was not started within 48 hours of admission, (b) the medical file could not be found or (c) hospitalisation in the ward was $<5$ days, due to discharge from the hospital or intensive care unit (ICU) admission during the first 120 inpatient hours, since the iSSC was not used in either the outpatient or citical care settings. Where a patient was eligible for the analysis for more than one hospitalisation during the study period, only data from the first hospitalisation were used.

The local ethical board (Kantonale Ethikkommission Aargau) approved this analysis, and due to the work's retrospective nature as a quality-control project and lack of patient interventions, waived the need for written informed consent.

\section{Glucose measurement and insulin treatment}

In all patients, glucose levels were recorded and insulin therapy was initiated as directed by the treating physician team according to the then-prevailing hospital insulin protocol, as that team judged to be clinically appropriate. Typically, glucose levels were measured 4 times daily, thrice before meals plus once before bed-time. Two-hour postprandial glucose levels were sometimes also measured, for applying correction insulin for hyperglycaemia.

According to the hospital pharmacy, during the inpatient stay, patients in both groups received mainly once or twice daily doses of long-acting insulin, generally Levemir ${ }^{\circ}$ (insulin detemir, Novo Nordisk, Bagsvaerd, Denmark) or, in some cases, Lantus ${ }^{\oplus}$ (insulin glargine, Sanofi, Paris, France), plus pre-prandial corrective doses of short-acting insulin, principally Humalog (insulin lispro, Lilly, Indianapolis, IN, USA) or NovoRapid ${ }^{\oplus}$ (insulin aspart, Novo Nordisk).

\section{iSSC}

In the iSSC group, patients were treated according to the insulin algorithm in Table 1. Briefly, this protocol incorporated three factors for dosing insulin: (a) latest glucose level, (b) amount of carbohydrate intake and (c) degree of insulin resistance. Patients were categorised according to one of 5 levels of insulin resistance. For a patient with the lowest insulin resistance, classified as level 1 , we assumed that 1 unit of insulin would be necessary to cover $10 \mathrm{~g}$ of carbohydrate intake and also reduce glucose levels by $2-3 \mathrm{mmol} / \mathrm{L}$. In resistance level 2 , the amount of insulin to reach these goals would be doubled. Insulin resistance classification was dynamic. Typically, patients would start at level 1 upon admission. In high-acuity patients, e.g., those with sepsis or elevated inflammatory analytes, who were expected to have high insulin resistance, or inpatients on higher pre-admission insulin doses, a higher starting insulin resistance classification was recommended. The insulin resistance classification was adjusted daily in stepwise fashion, upwards if insulin doses failed to correct hyperglycaemic glucose levels or even failed to stop continuing glucose increases, and downwards if insulin doses were associated with hypoglycaemic glucose concentrations.

\section{SSC (Historical control protocol)}

The SSC algorithm called for a set amount of insulin to be administered based only on the patient's latest blood glucose level, without considering the timing or carbohydrate content of meals or snacks, the pre-admission insulin regimen, or the degree of insulin resistance.

With both the iSSC and the SSC, the treating physician team

\begin{tabular}{|c|c|c|c|c|c|c|c|}
\hline \multicolumn{8}{|c|}{ A. Rapid insulin in dose to correct increased blood glucose (Target level 5.5-7.0 mmol/L) } \\
\hline & \multicolumn{7}{|c|}{ Monitoring and insulin injection } \\
\hline Glucose control & \multicolumn{2}{|c|}{4 times per day } & \multicolumn{2}{|c|}{ every 4 hours } & \multicolumn{3}{|c|}{ every 2 hours } \\
\hline Glucose range (mmol/L) & 4.1-7.0 & 7.1-9.0 & $9.1-13.0$ & 13.1-16.0 & $16.1-19.0$ & $19.1-21.0$ & $>21.0$ \\
\hline Insulin units & 0 & 1 & 2 & 3 & 4 & 5 & 6 \\
\hline
\end{tabular}

\begin{tabular}{|c|c|c|c|}
\hline \multicolumn{2}{|c|}{ B. Rapid insulin dose to cover meal carbohydrate intake } \\
\hline Meal size & \multicolumn{2}{|c|}{ Insulin units (carbohydrates) } \\
\hline & breakfast & lunch & \multicolumn{2}{|c|}{ dinner } \\
\hline $1200 \mathrm{kcal}$ & $3(30)$ & $3(30)$ & $3(30)$ \\
\hline $1600 \mathrm{kcal}$ & $4(40)$ & $4(40)$ & $4(40)$ \\
\hline $2000 \mathrm{kcal}$ & $5(50)$ & $5(50)$ & $5(50)$ \\
\hline $2400 \mathrm{kcal}$ & $6(60)$ & $6(60)$ & $6(60)$ \\
\hline
\end{tabular}

Total rapid insulin dose $(A+B)$ * resistance factor = total insulin units

Generally, start with resistance factor 1 . In high-acuity patients (e.g., those with sepsis or systemic inflammation), patients with corticosteroid treatment or in patients on high pre-admission insulin doses, a higher starting insulin resistance factor may be considered.

Table 1: "Improved" sliding scale insulin protocol incorporating nutritional factors and insulin resistance as well as glucose levels for insulin dosing. 
Citation: Schuetz P, Braun N, Germann S, Baumgartner A, Mueller B (2014) Incorporation of Nutritional Factors and Insulin Resistance into Insulin Sliding Scales in the Non-Critical Care Inpatient Setting: A "Before/After" Study. J Diabetes Metab 2: 325. doi:10.4172/2155-6156.1000325

Page 3 of 6

were permitted to modify or disregard the protocol based on clinical judgment.

\section{Endpoints}

The primary endpoints were 1) the mean glucose level over the first 120 inpatient hours and 2) the percentage of that time at euglycaemic glucose levels (3.8-7.0 $\mathrm{mmol} / \mathrm{L})$. The mean glucose concentration over the first 120 hours was calculated using all available measured glucose concentrations within that period and assuming a linear trend between those measurements.

Secondary endpoints included occurrence during the first 120 hours of any episode of hypoglycaemia, subcategorised as mild (blood glucose $2.5-3.8 \mathrm{mmol} / \mathrm{L})$ or severe $(<2.5 \mathrm{mmol} / \mathrm{L})$, hospital length-ofstay, all-cause mortality after the first 120 inpatient hours, and ICU admission during the hospital stay.

\section{Statistical analysis}

To describe the populations, values are expressed as means with standard deviations (SDs) and frequencies as percentages, counts or both, as appropriate. We investigated differences in endpoints between the iSSC group and the historical control group with linear and logistic regression analysis adjusted for important potential confounders, specifically diabetes type, time since diabetes diagnosis, BMI, presence of dyslipidaemia, admission blood glucose concentration, preadmission chronic therapy (metformin, insulin), and main hospital diagnosis (LRTI or acute cardiac condition).

In a second step, we investigated factors potentially associated with hypoglycaemia in the overall study population using logistic regression analysis and stepwise selection procedures at the $\mathrm{p}<0.2$ limit. All analyses were performed with Stata 12 (StataCorp, College Station, TX, USA).

\section{Results}

\section{Study participants}

Altogether, 215 inpatients with diabetes, almost always (207/215, 96.9\%) type II, were eligible and included into this analysis; the principal cause of hospitalisation was LRTI in 102 patients (47.4\%) and an acute cardiac condition in 113 (52.6\%). In total, 128 patients (59.5\%) were in the iSSC group and 87 (40.5\%) in the historical control group;

Table 2 shows baseline characteristics for the overall study sample and by treatment group. The two cohorts were well balanced regarding nearly all tested socio-demographic and metabolic variables and factors related to diabetes and other comorbidities. Yet, the iSSC group had a significantly greater prevalence of dyslipidaemia and a significantly shorter interval since diabetes diagnosis than did the SSC patients.

\begin{tabular}{|c|c|c|c|c|}
\hline Variable $^{a}$ & Overall $(n=215)$ & SSC group $(n=87)$ & iSSC group $(n=128)$ & $\mathrm{p}$ \\
\hline \multicolumn{5}{|l|}{ Demographics } \\
\hline Age (years) & $69.4( \pm 12.7)$ & $69.0( \pm 13.1)$ & $69.6( \pm 12.4)$ & 0.73 \\
\hline Male sex & $64.7 \%(139)$ & $70.1 \%(61)$ & $60.9 \%(78)$ & 0.17 \\
\hline Weight (kg) & $84.3( \pm 16.1)$ & $85.8( \pm 17.3)$ & $83.4( \pm 15.2)$ & 0.29 \\
\hline Body mass index $\left(\mathrm{kg} / \mathrm{m}^{2}\right)$ & $29.5( \pm 5.2)$ & $29.9( \pm 5.6)$ & $29.3( \pm 4.9)$ & 0.40 \\
\hline Obesity (BMI>30) & $77.2 \%(166)$ & $77.0 \%(67)$ & $77.3 \%(99)$ & 0.96 \\
\hline \multicolumn{5}{|l|}{ Metabolic variables } \\
\hline Diabetes Type 1 & $3.7 \%(8)$ & $4.6 \%(4)$ & $3.1 \%(4)$ & 0.56 \\
\hline Diabetes Type 2 & $96.3 \%(207)$ & $95.4 \%(83)$ & $96.9 \%(124)$ & 0.58 \\
\hline $\begin{array}{l}\text { Time since diabetes } \\
\text { diagnosis (months) }\end{array}$ & $12.4( \pm 10.3)$ & $14.9( \pm 11.2)$ & $11.1( \pm 9.6)$ & 0.03 \\
\hline Admission HbA1c (\%) & $7.8( \pm 1.4)$ & $7.9( \pm 1.6)$ & $7.8( \pm 1.3)$ & 0.66 \\
\hline Initial blood glucose level (mmol/L) & $11.5( \pm 4.8)$ & $12.0( \pm 5.9)$ & $11.1( \pm 4)$ & 0.22 \\
\hline Dyslipidaemia & $50.7 \%(109)$ & $42.5 \%(37)$ & $56.3 \%(72)$ & 0.04 \\
\hline \multicolumn{5}{|l|}{ Comorbidities and smoking status } \\
\hline Hypertension & $75.8 \%(163)$ & $78.2 \%(68)$ & $74.2 \%(95)$ & 0.57 \\
\hline Chronic renal failure & $34.4 \%(74)$ & $31.0 \%(27)$ & $36.7 \%(47)$ & 0.39 \\
\hline Malignancy & $16.3 \%(35)$ & $16.1 \%(14)$ & $16.4 \%(21)$ & 0.95 \\
\hline Dementia & $8.8 \%(19)$ & $12.67 \%(11)$ & $6.3 \%(8)$ & 0.11 \\
\hline Current or former smoker & $55.8 \%(120)$ & $58.6 \%(15)$ & $53.9 \%(69)$ & 0.42 \\
\hline \multicolumn{5}{|l|}{ Medication on admission } \\
\hline Metformin & $45.6 \%(98)$ & $44.8 \%(39)$ & $46.1 \%(59)$ & 0.91 \\
\hline Sulfonylurea & $32.6 \%(70)$ & $31.0 \%(27)$ & $33.6 \%(43)$ & 0.76 \\
\hline Other oral anti-diabetic agents & $18.1 \%(39)$ & $13.8 \%(12)$ & $21.1 \%(27)$ & 0.22 \\
\hline Any insulin & $30.7 \%(66)$ & $29.9 \%(26)$ & $31.3 \%(40)$ & 0.83 \\
\hline Total daily insulin dose, $U$ & $33.1( \pm 19.4)$ & $29.1( \pm 19.3)$ & $35.4( \pm 19.3)$ & 0.25 \\
\hline Steroid treatment & $16.7 \%(36)$ & $17.2 \%(15)$ & $16.4 \%(21)$ & 0.86 \\
\hline \multicolumn{5}{|l|}{ Hospital variables } \\
\hline $\begin{array}{l}\text { Cause of hospitalisation } \\
\text { LRTI } \\
\text { Acute cardiac condition }\end{array}$ & $\begin{array}{l}47.4 \%(102) \\
52.6 \%(113)\end{array}$ & $\begin{array}{l}50.6 \%(44) \\
49.4 \%(43)\end{array}$ & $\begin{array}{l}45.3 \%(58) \\
54.7 \%(70)\end{array}$ & 0.49 \\
\hline
\end{tabular}

BMI: Body-Mass Index; LRTI: Lower Respiratory Tract Infection; SD: Standard Deviation

aVariables are expressed as percentage of (number in) study sample or group or as mean [ \pm SD]

Table 2: Baseline characteristics in the overall study sample and according to treatment group. 


\section{Outcomes}

There were no serious diabetes-related complications (coma, cause-specific death) in either treatment group. Table 3 presents adjusted regression coefficients or odds ratios for outcomes of interest for patients in the iSSC relative to historical controls. Compared to the historical control group, patients in the iSSC group consistently had a trend toward better glucose control over the first 120 inpatient hours (mean blood glucose, hyperglycaemic or hypoglycaemic episodes, glucose variability, incidence of mild or severe hypoglycaemia), as evidenced by negative adjusted regression coefficients or adjusted odds ratios <1.0, but without reaching statistical significance. Additionally, iSSC patients had consistently better hospital outcomes, as reflected by a negative adjusted regression coefficient for hospital length-of-stay and odds ratios $<1.0$ for inhospital mortality or ICU admission, but again, none of these differences attained statistical significance.

\section{Factors associated with hypoglycaemia}

Table 4 displays the results of a logistic regression analysis investigating factors potentially associated with hypoglycaemia (blood glucose $<3.8 \mathrm{mmol} / \mathrm{L}$ at any time within the first 120 inpatient hours). Chronic metformin therapy at the time of admission and male gender were associated with significantly lower probability, and a longer interval since diabetes diagnosis and LRTI rather than an acute cardiac condition as the cause of hospitalisation were associated with significantly higher probability of hypoglycaemia.

\section{Discussion}

Physiological glucose ranges in patients with systemic infections and acute cardiac conditions remain ill-defined. However, recent observational data from both critical care units [28-30] and noncritical care wards $[11,31,32]$ worldwide include strong correlations of

\begin{tabular}{|c|c|c|}
\hline Outcome & $\begin{array}{l}\text { Adjusted }^{\mathrm{a}} \text { regression } \\
\text { coefficient or odds ratio } \\
(95 \% \mathrm{Cl})\end{array}$ & p \\
\hline \multicolumn{3}{|c|}{ Mean blood glucose during the first 120 hours } \\
\hline Mean blood glucose (mmol/L) & $-0.53(-1.28,0.22)^{b}$ & 0.163 \\
\hline \multicolumn{3}{|c|}{ Glycaemic range during the first 120 hours } \\
\hline Hyperglycaemic range $(7.0 \mathrm{mmol} / \mathrm{L})$ & $-5.73(-14.8,3.35)^{b}$ & 0.214 \\
\hline Hypoglycaemic range $(4.0 \mathrm{mmol} / \mathrm{L})$ & $-0.82(0.79,-2.43)^{b}$ & 0.313 \\
\hline \multicolumn{3}{|c|}{ Glucose variability during the first 120 hours } \\
\hline Variability during the first 120 hours & $-1.28(1.27,-3.83)^{b}$ & 0.322 \\
\hline \multicolumn{3}{|l|}{ Hypoglycaemia during the first 120 hours } \\
\hline Mild hypoglycaemia $(2.5-3.8 \mathrm{mmol} / \mathrm{L})$ & $0.59(0.27,1.29)^{\mathrm{c}}$ & 0.187 \\
\hline Severe hypoglycaemia $(<2.5 \mathrm{mmol} / \mathrm{L})$ & $0.33(0.03,3.75)^{\mathrm{c}}$ & 0.375 \\
\hline \multicolumn{3}{|l|}{ Hospital outcomes } \\
\hline Ward length-of-stay (days) & $-1.62(-3.63,0.40)^{b}$ & 0.114 \\
\hline In-hospital mortality & $0.39(0.04,3.58)^{c}$ & 0.406 \\
\hline Transfer to ICU & $0.35(0.03,3.99)^{c}$ & 0.397 \\
\hline
\end{tabular}

BMI: Body-Mass Index; ICU: Intensive Care Unit; LRTI: Lower Respiratory Tract Infection

a Adjusted for diabetes type and time since diabetes diagnosis, BMI, presence of dyslipidaemia, admission blood glucose concentration, chronic therapy at admission (metformin, insulin), and main hospital diagnosis (LRTI or acute cardiac condition).

${ }^{\mathrm{b}}$ Adjusted regression coefficient.

${ }^{c}$ Adjusted odds ratio.

Table 3: Primary and secondary outcomes: adjusted regression coefficient or odds ratio for iSSC group relative to historical control group

\begin{tabular}{|l|c|c|}
\hline Outcome & $\begin{array}{c}\text { Adjusted odds } \\
\text { ratio (95\%Cl) }\end{array}$ & p value \\
\hline Pre-existing metformin therapy & $0.16(0.04,0.66)$ & 0.011 \\
\hline Duration since diabetes onset (per year) & $1.07(1.01,1.12)$ & 0.012 \\
\hline LRTI & $4.27(1.28,14.27)$ & 0.018 \\
\hline Male gender & $0.26(0.08,0.88)$ & 0.03 \\
\hline Glucose level upon hospital admission (per mmol/L) & $1.08(0.96,1.22)$ & 0.189 \\
\hline
\end{tabular}

LRTI: Lower Respiratory Tract Infection

Table 4: Association of tested factors with hypoglycaemia.

initial and average inhospital glucose levels, hypoglycaemia, or glucose variability with in-hospital mortality and other adverse outcomes. These observations highlight the desirability of improving glucose control in this setting. Importantly, randomised trials have further strengthened the inverse association of hypoglycaemia and increased mortality [29,30]. These observations led to the current Endocrine Society Clinical Practice Guidelines recommendation to target a premeal glucose level of $<140 \mathrm{mg} / \mathrm{dL}(<7.8 \mathrm{mmol} / \mathrm{L})$ and a random level of $<180 \mathrm{mg} / \mathrm{dL}(<10.0 \mathrm{mmol} / \mathrm{L})$ for most inpatients with non-critical illness [11]. However, the guidelines also note that for inpatients at elevated hypoglycaemia risk, a higher target blood glucose range may be reasonable.

Despite these findings and recommendations, safe, efficient protocols to achieve these targets with s.c. insulin injections outside the intensive care setting are not well-established.

In an attempt to optimise in-house insulin protocols to correct hyperglycaemia and avoid hypoglycaemia and glucose variability, respectively, in general medical wards, we recently completed a twocentre, randomised controlled trial to test the safety and efficacy of a more sophisticated and rigorous insulin protocol (the iSSC), incorporating insulin resistance and carbohydrate intake, as well as serum glucose levels [27]. The study found improved, early and sustained glycaemic control with the new protocol in non-critically ill patients admitted to general medical wards, without apparent safety concerns. However, small sample size $(\mathrm{N}=130)$ and considerable patient heterogeneity regarding underlying illness may have limited that study's external validity.

We, therefore, conducted the present retrospective analysis of the "real world" experience of another Swiss hospital with everyday use of a protocol largely similar to that applied in the randomised study in a larger, more defined patient cohort. We found that relative to an algorithm guided only by blood glucose levels, the iSSC was associated with consistent improvement of mean estimates across glucose control and more general clinical outcomes. Although the confidence intervals overlapped and did not attain statistical significance, the consistency of our findings further suggests the potential benefits of incorporating nutritional and insulin resistance factors into insulin protocols for glucose control.

We also investigated potential baseline predictors of hypoglycaemia. We identified both protective factors, i.e., male gender, chronic metformin therapy, and risk factors, i.e., longer diabetes duration, LRTI diagnosis and higher initial glucose levels, which may merit further study as additional inputs to SSC protocols, and more generally speaking.

Limitations of the present analysis merit consideration. First, the analysis relied on retrospectively collected data from everyday practice; no information was available on the extent of physician adherence to either the iSSC or the SSC. Since physicians were allowed to depart 
Citation: Schuetz P, Braun N, Germann S, Baumgartner A, Mueller B (2014) Incorporation of Nutritional Factors and Insulin Resistance into Insulin Sliding Scales in the Non-Critical Care Inpatient Setting: A "Before/After" Study. J Diabetes Metab 2: 325. doi:10.4172/2155-6156.1000325

Page 5 of 6

from both protocols based on clinical judgment, the dearth of such data represents a potentially appreciable factor confounding interpretation of our results. Indeed, one can speculate that the more lax adherence to the iSSC likely to occur in everyday practice as opposed to a randomised controlled study may account for the lack of statistical significance of the glucose control and other benefits in our cohort. Second, due to the "before/after" design, groups may not be well-balanced. Nonetheless, of numerous studied characteristics, our cohorts differed only in two, prevalence of dyslipidaemia (higher in the iSSC group) and time since diabetes diagnosis (longer in historical controls). Additionally, we used multivariate regression models to adjust for a variety of potentially relevant baseline patient and disease characteristics. Still, residual confounding is possible. Third, the power of our sample size was low, which also may explain the lack of significance regarding our primary and secondary endpoints. Lastly, our study provides a suggestion but no statistically significant evidence whether improved glucose control results in better patient outcomes. However, in light of the consistency of our findings and of recent data about the association of hyperglycaemia and adverse outcomes, it seems reasonable to postulate that improvement of glucose control through more sophisticated insulin algorithms may also result in better patient outcomes.

In conclusion, our analysis suggests borderline benefits of incorporating nutritional factors and insulin resistance factors into insulin protocols to improve glucose control and reduce the risk of hypoglycaemia. Larger prospective randomised controlled trials should look into the benefits of more rigorous insulin protocols to improve inpatient management outside critical care.

\section{Acknowledgements}

We thank all participating patients and physicians. We also thank Robert Marlowe for editing this manuscript. Novo Nordisk provided an unrestricted research grant for data abstraction. None of the authors has any other conflict of interest to declare.

\section{References}

1. Cooper MS, Stewart PM (2003) Corticosteroid insufficiency in acutely ill patients. N Engl J Med 348: 727-734.

2. Foo K, Cooper J, Deaner A, Knight C, Suliman A, et al. (2003) A single serum glucose measurement predicts adverse outcomes across the whole range of acute coronary syndromes. Heart 89: 512-516.

3. Malmberg K (1997) Prospective randomised study of intensive insulin treatment on long term survival after acute myocardial infarction in patients with diabetes mellitus. DIGAMI (Diabetes Mellitus, Insulin Glucose Infusion in Acute Myocardial Infarction) Study Group. BMJ 314: 1512-1515

4. Malmberg K, Norhammar A, Wedel H, Rydén L (1999) Glycometabolic state at admission: important risk marker of mortality in conventionally treated patients with diabetes mellitus and acute myocardial infarction: long-term results from the Diabetes and Insulin-Glucose Infusion in Acute Myocardial Infarction (DIGAMI) study. Circulation 99: 2626-2632.

5. Malmberg K, Rydén L, Efendic S, Herlitz J, Nicol P, et al. (1995) Randomized trial of insulin-glucose infusion followed by subcutaneous insulin treatment in diabetic patients with acute myocardial infarction (DIGAMI study): effects on mortality at 1 year. J Am Coll Cardiol 26: 57-65.

6. Malmberg K, Ryden L, Hamsten A, Herlitz J, Waldenstrom A, et al. (1996) Effects of insulin treatment on cause-specific one-year mortality and morbidity in diabetic patients with acute myocardial infarction. DIGAMI Study Group. Diabetes Insulin-Glucose in Acute Myocardial Infarction. Eur Heart J 17: 13371344.

7. Malmberg KA, Efendic S, Rydén LE (1994) Feasibility of insulin-glucose infusion in diabetic patients with acute myocardial infarction. A report from the multicenter trial: DIGAMI. Diabetes Care 17: 1007-1014.

8. der Voort PH, Feenstra RA, Bakker AJ, Heide L, Boerma EC, et al. (2006) Intravenous glucose intake independently related to intensive care unit and hospital mortality: an argument for glucose toxicity in critically ill patients. Clin Endocrinol (Oxf) 64: 141-145.
9. van den Berghe $G$, Wouters $P$, Weekers $F$, Verwaest $C$, Bruyninckx $F$, et al. (2001) Intensive insulin therapy in critically ill patients. N Engl J Med 345: 13591367.

10. Van den Berghe G, Wilmer A, Hermans G, Meersseman W, Wouters PJ, et al. (2006) Intensive insulin therapy in the medical ICU. N Engl J Med 354: 449-461.

11. Umpierrez GE, Hellman R, Korytkowski MT, Kosiborod M, Maynard GA, et al (2012) Management of hyperglycemia in hospitalized patients in non-critical care setting: an Endocrine Society clinical practice guideline. J Clin Endocrinol Metab 97: 16-38.

12. Baird TA, Parsons MW, Phan T, Butcher KS, Desmond PM, et al. (2003) Persistent poststroke hyperglycemia is independently associated with infarct expansion and worse clinical outcome. Stroke 34: 2208-2214.

13. Plank J, Blaha J, Cordingley J, Wilinska ME, Chassin LJ, et al. (2006) Multicentric, randomized, controlled trial to evaluate blood glucose control by the model predictive control algorithm versus routine glucose management protocols in intensive care unit patients. Diabetes Care 29: 271-276.

14. Scott JF, Robinson GM, French JM, O'Connell JE, Alberti KG, et al. (1999) Glucose potassium insulin infusions in the treatment of acute stroke patients with mild to moderate hyperglycemia: the Glucose Insulin in Stroke Trial (GIST). Stroke 30: 793-799.

15. Buchs AE, Bloemers R, Shiloah E, Horowitz M, Aminov S, et al. (2009) Feasibility and safety of multiple daily insulin injections in general medicine wards. Eur J Intern Med 20: 435-439.

16. Meyer C, Boron A, Plummer E, Voltchenok M, Vedda R (2010) Glulisine versus human regular insulin in combination with glargine in noncritically ill hospitalized patients with type 2 diabetes: a randomized double-blind study. Diabetes Care 33: $2496-2501$

17. Patel GW, Roderman N, Lee KA, Charles MM, Nguyen D, et al. (2009) Sliding scale versus tight glycemic control in the noncritically ill at a community hospital. Ann Pharmacother 43: 1774-1780.

18. Umpierrez GE, Smiley D, Jacobs S, Peng L, Temponi A, et al. (2011) Randomized study of basal-bolus insulin therapy in the inpatient management of patients with type 2 diabetes undergoing general surgery (RABBIT 2 surgery). Diabetes Care 34: 256-261.

19. Umpierrez GE, Smiley D, Zisman A, Prieto LM, Palacio A, et al. (2007) Randomized study of basal-bolus insulin therapy in the inpatient management of patients with type 2 diabetes (RABBIT 2 trial). Diabetes Care 30: 2181-2186.

20. Mizock BA (2003) Blood glucose management during critical illness. Rev Endocr Metab Disord 4: 187-194.

21. Festa A, D'Agostino R Jr, Williams K, Karter AJ, Mayer-Davis EJ, et al. (2001) The relation of body fat mass and distribution to markers of chronic inflammation. Int J Obes Relat Metab Disord 25: 1407-1415.

22. Forouhi NG, Sattar N, McKeigue PM (2001) Relation of C-reactive protein to body fat distribution and features of the metabolic syndrome in Europeans and South Asians. Int J Obes Relat Metab Disord 25: 1327-1331.

23. Jayagopal V, Kilpatrick ES, Jennings PE, Holding S, Hepburn DA, et al. (2004) The biological variation of sex hormone-binding globulin in type 2 diabetes implications for sex hormone-binding globulin as a surrogate marker of insulin resistance. Diabetes Care 27: 278-280.

24. Laaksonen DE, Niskanen L, Punnonen K, Nyyssönen K, Tuomainen TP, et al (2004) Testosterone and sex hormone-binding globulin predict the metabolic syndrome and diabetes in middle-aged men. Diabetes Care 27: 1036-1041.

25. Lee WY, Park JS, Noh SY, Rhee EJ, Sung KC, et al. (2004) C-reactive protein concentrations are related to insulin resistance and metabolic syndrome as defined by the ATP III report. Int J Cardiol 97: 101-106.

26. Wajchenberg BL (2000) Subcutaneous and visceral adipose tissue: their relation to the metabolic syndrome. Endocr Rev 21: 697-738.

27. Thomann R, Schuetz, Muller B, Thomke S, Schoenenberger R, et al. (2013) Evaluation of an algorithm for intensive subcutaneous insulin therapy in noncritically ill hospitalised patients with hyperglycaemia in a randomised controlled trial. Swiss Med Wkly 143: w13808.

28. Krinsley JS, Egi M, Kiss A, Devendra AN, Schuetz P, et al. (2013) Diabetic status and the relation of the three domains of glycemic control to mortality in critically ill patients: an international multicenter cohort study. Crit Care 17: R37. 
Citation: Schuetz P, Braun N, Germann S, Baumgartner A, Mueller B (2014) Incorporation of Nutritional Factors and Insulin Resistance into Insulin Sliding Scales in the Non-Critical Care Inpatient Setting: A "Before/After" Study. J Diabetes Metab 2: 325. doi:10.4172/2155-6156.1000325

Page 6 of 6

29. NICE-SUGAR Study Investigators, Finfer S, Liu B, Chittock DR, Norton R, et al. (2012) Hypoglycemia and risk of death in critically ill patients. N Engl J Med 367: 1108-1118.

30. NICE-SUGAR Study Investigators, Finfer S, Chittock DR, Su SY, Blair D, et al. (2009) Intensive versus conventional glucose control in critically ill patients. N

\section{Engl J Med 360: 1283-1297.}

31. Schuetz P, Castro P, Shapiro NI (2011) Diabetes and sepsis: preclinical findings and clinical relevance. Diabetes Care 34: 771-778.

32. Schuetz P, Kennedy M, Lucas JM, Howell MD, Aird WC, et al. (2012) Initial management of septic patients with hyperglycemia in the noncritical care inpatient setting. Am J Med 125: 670-678. 